\title{
Correspondance
}

\section{Social and economic justice: the road to health}

Tisagree with Prabhat Jha and James 1 Lavery $^{1}$ that technological advances, rather than improvements in income and education, have been responsible for improvements in health care in the developing world.

Sri Lanka and the state of Kerala in India have made rapid advances in health care through their investment in primary care and education and measures to reduce social and health inequities. Despite a civil war, Sri Lanka has the best health indicators in South East Asia, with average life expectancy of 73 years, infant mortality of 16 per 1000 and maternal mortality of 30 per 100000 live births. ${ }^{2}$ This country does not have a single MRI scanner in the public sector, which highlights an emphasis on primary care. Similarly, Kerala has achieved health and demographic indicators far ahead of Indian national averages. ${ }^{2}$

The greatest health hazard is in fact the economic gap between the rich and the poor. According to population health studies, the primary determinant of health within a country is societal structure, especially the degree of hierarchy as measured by income distribution. ${ }^{3}$ Not only is the health of a population related to income, but at any given level of overall economic development for a country or a region within a country, the populations of countries and regions with smaller gaps between rich and poor are, in general, healthier than the populations of countries and regions where the gap is larger. ${ }^{4} \mathrm{~A}$ case in point is the United States, which spent the most on health care but ranked only 26th in life expectancy in 2003; Japan was the top country in this ranking. ${ }^{5}$ Among the developed countries, the United States has the greatest gap between rich and poor, whereas Japan has the smallest gap.

The road to health for developing countries lies not in blindly emulating the West in terms of sophisticated medical care but in making sound in- vestments in primary care and education as well as promoting social and economic justice.

\section{Sonal Singh \\ Unity Health System \\ Rochester, NY}

\section{References}

1. Jha P, Lavery JV. Evidence for global health [editorial]. CMA7 2004;170(11):1687-8.

2. Bhutta $Z$, Nundy $S$, Abbasi K. Is there hope for South Asia? [editorial]. BM7 2004;328(7443):777-8.

3. Bezruchka S. Societal hierarchy and the health Olympics [editorial]. CMA7 2001;164(12):1701-3.

4. Van Doorslaer E, Wagstaff A, Bleichrodt H, Calonge S, Gerdtham UG, Gerfin M, et al. Income-related inequalities in health: some international comparisons. 7 Health Econ 1997;16(1): 93-112.

5. Population Health Forum. U.S.A. richest nation. Big gap civilization. Sickest population [online]. Seattle: University of Washington, School of Public Health and Community Medicine; 2003. Available: http://depts.washington.edu/eqhlth /index.htm (accessed 2004 Sept 09).

DOI:10.1503/cmaj.1040922

\section{[The authors respond:]}

W Te suspect that Sonal Singh has overestimated the extent to which our views differ from his about the determinants of health and health disparities globally. In our commentary ${ }^{1}$ we state that the chief reason for health improvements in developing countries in the second half of the 20th century - for which we cite reductions in mortality rates as a main indicator — has been a wide range of technological advances, rather than improvements in income or education.

However, our definition of technological advances is not "sophisticated medical care." Rather, such advances include widely practicable drugs, vaccines, treatment and prevention protocols, public health campaigns (such as the eradication of smallpox) and personal knowledge (such as the hazards of smoking). ${ }^{2}$ All of these technologies arose from research.

Singh points out that there are numerous determinants of health, including distal factors such as income and education. However, income and education matter less than we might first guess. Easterlin has written probably the best review of historical improvements in health and the role of income and education. ${ }^{3}$ He concluded that focused public health initiatives were responsible for most of the declines in mortality rates in recent human history. A more recent review also found that, if anything, the linkages of health to income were greater than the linkages of income to health. ${ }^{4}$

Moreover, a determinant of health may not be an intervention. Yes, there are examples such as Sri Lanka and Kerala where good health has been achieved at low levels of expenditure. However, a careful comparison across 2 dozen Indian states found that differences in income growth (or education) over the previous 2 decades did not appear to account for variation in mortality rates for children under 5 years of age. ${ }^{5}$ Rather, coverage with an extended program of vaccination, treatment of acute respiratory infection in a medical facility and use of oral rehyhdration therapy for diarrhea showed a clear gradient from worst- to best-performing states. ${ }^{6}$

Taken to an extreme (which Singh does not do), the suggestion that we should wait for equality and social justice (desirable as these are for other reasons) to reduce mortality and disability strikes us as somewhere between romantic and nihilistic. The chief challenge today is to control some of the major diseases worldwide. Such control requires solid public health action, implementing currently known effective interventions, and research to generate new tools.

\section{Prabhat Jha}

Centre for Global Health Research

James V. Lavery

Centre for Global Health Research and

Inner City Health Research Unit

St. Michael's Hospital

University of Toronto

Toronto, Ont.

\section{References}

1. Jha P, Lavery JV. Evidence for global health [editorial.] CMA7 2004;170(11):1687-8.

2. Jha P, Mills A, Hanson K, Kumaranayake L, 
Conteh L, Kurowski C, et al. Improving the health of the global poor. Science 2002;295:2036-9.

3. Easterlin RA. How beneficent is the market? A look at the modern history of mortality. Eur Rev Econ Hist 1999;3(3):257-94.

4. Deaton A. Health, inequality, and economic development. Paper no. WG1:3 of CMH Working Paper Series. [place unknown]: Commission on Macroeconomics and Health; 2001 May. Available: www.cmhealth.org/docs/wg1_paper3.pdf (accessed 2004 Sep 8)

5. Measham AR, Rao KD, Jamison DT, Wang J, Singh A. The performance of India and Indian states in reducing infant mortality and fertility, 1975-1990. Econ Polit Wkly 1999;34(22):1359-67.

6. Jha P. Avoidable mortality in India: past progres and future prospects. Natl Med 7 India 2002;15 (Suppl 1):32-6.

DOI:10.1503/cmaj.1041238

\section{Seeking clarification of osteoporosis guidelines}

$\mathrm{T}$ he recent statement of the Canadian Task Force on Preventive Health Care regarding prevention of osteoporosis and osteoporotic fractures in postmenopausal women ${ }^{1}$ contains some confusing information. One example is the statement that "Although there is no direct evidence that screening reduces fractures, there is good evidence that screening is effective in identifying postmenopausal women with low bone mineral density and that treating osteoporosis can reduce the risk of fractures in this population." This wording appears to have been chosen to obfuscate the meaning, since low bone mineral density, particularly in the younger population, does not strongly correlate with fracture risk or osteoporosis. ${ }^{2,3}$

Other parts of the recommendation statement do not appear particularly practical. For example, the algorithm shown in Fig. 1 of the article ${ }^{1}$ suggests that all women 65 years of age or older should undergo repeat dual-energy $\mathrm{x}$ ray absorptiometry (DEXA) every 1 to 2 years, regardless of the result of initial DEXA (even if that result is normal). Admittedly, this agrees with the guidelines of the US Preventive Services Task Force ${ }^{4}$ and the Osteoporosis Society of Canada, ${ }^{2}$ but what does it mean for those of us providing primary care? Should we in fact send all of our female patients over age 65 , including those in rest homes, for DEXA screening? Would it not be adequate to suggest to women in this age group that they try to exercise regularly and take adequate amounts of vitamin D and calcium?

Also of great concern are the potential medicolegal implications if clinicians do not follow guidelines developed by authoritative bodies such as the Task Force.

Do the CMA7 editors accept guidelines and protocols produced by distinguished Canadian associations (often sponsored by drug companies) without the benefit of peer review or editing?

\section{John Sehmer}

Clinical Assistant Professor

University of British Columbia

Vancouver, BC

\section{References}

1. Cheung AM, Feig DS, Kapral M, Diaz-Granados, Dodin S and The Canadian Task Force on Preventive Health Care. Prevention of osteoporosis and osteoporotic fractures in postmenopausal women: recommendation statement from the Canadian Task Force on Preventive Health Care. CMAf 2004;170(11):1665-7.

2. Brown JP, Josse RG, for the Scientific Advisory Council of the Osteoporosis Society of Canada. 2002 clinical practice guidelines for the diagnosis and management of osteoporosis in Canada. CMA7 2002:167(10 Suppl):S1-34.

3. Moynihan R, Heath I, Henry D. Selling sickness: the pharmaceutical industry and disease mongering [editorial]. BM7 2002;324:886-91.

4. US Preventive Services Task Force. Screening for osteoporosis in postmenopausal women: recommendations and rationale. Ann Intern Med 2002;137:526-8

Competing interests: None declared.

DOI:10.1503/cmaj.1040991

$\mathrm{T}$ he recommendation statement from the Canadian Task Force on Preventive Health Care about preventing osteoporosis and osteoporotic fractures in postmenopausal women ${ }^{1}$ mentions oral pamidronate as a second-line drug choice. However, to the best of my knowledge, oral pamidronate is not available in Canada.

\section{Mario L. de Lemos}

British Columbia Cancer Agency

Vancouver, BC

\section{Reference}

1. Cheung AM, Feig DS, Kapral M, Diaz-Granados N, Dodin S and The Canadian Task Force on Preventive Health Care. Prevention of osteo- porosis and osteoporotic fractures in postmenopausal women: recommendation statement from the Canadian Task Force on Preventive Health Care. CMA7 2004;170(11):1665-7.

Competing interests: None declared.

DOI:10.1503/cmaj.1040953

\section{[The lead author and the chair of the Task Force respond:]}

7 he recent recommendation statement concerning the prevention of osteoporosis and osteoporotic fractures in postmenopausal women ${ }^{1}$ was developed after a detailed process of identifying the appropriate analytic framework, systematically reviewing the literature, discussing the evidence at multiple Task Force meetings and subjecting the statement to 2 levels of peer review (internal peer review within the Task Force and external peer review organized by the Task Force).

On the basis of our analytic framework and the evidence available, we concluded that there is no direct evidence that screening reduces fractures. In other words, there were no acceptable randomized controlled trials that directly evaluated routine screening linked to treatment compared with usual care. However, there is evidence that screening is effective in identifying postmenopausal women with osteoporosis. There is also evidence that treating osteoporosis can reduce the risk of fractures in postmenopausal women. Because the evidence that supports fracture reduction through screening is therefore indirect, our overall recommendation was grade $\mathrm{B}$, rather than grade $\mathrm{A}$. Currently, there is much controversy as to what the treatment threshold should be. Most experts agree that postmenopausal women with osteoporosis ( $T$ score at or below -2.5) should be treated with pharmacologic therapies, because there is good to fair evidence from randomized controlled trials that such treatment will reduce osteoporotic fractures in this population. Some of these trials have included women with $T$ scores between -2.0 and -2.5 .

There is a strong correlation be- 